\title{
Articles of Significant Interest in This Issue
}

Modulation of Response Regulator Phosphorylation and Dephosphorylation Kinetics by Variable Residues

Protein phosphorylation and dephosphorylation reactions used for signal transduction must be fast enough to convey environmental changes in real time. Straughn et al. (e00089-20) describe how changes at two positions vary the autophosphorylation rate constant of the CheY response regulator over a 100 -fold range by altering protein conformation. They also summarize how five variable positions modulate CheY autophosphorylation and autodephosphorylation rate constants over three orders of magnitude each. The distributions of variable residue combinations across response regulator subfamilies suggest that distinct mechanisms associated with different variable positions allow reaction rates to be tuned independently during evolution for diverse biological purposes.

Intra- and Extracellular Galactosylation of Bacillus anthracis Secondary Cell Wall Polysaccharide

Bacillus anthracis elaborates a secondary cell wall polysaccharide (SCWP) of trisaccharide repeat units. Modifications of repeat units impact envelope assembly, cell division, and interactions with viruses and infected hosts. Château et al. (e00191-20) propose that $\beta$-Gal modification of SWCP is mediated by cytosolic GtsE while $\alpha$-Gal modification is carried out by GtsA, GtsB, and GtsC on the trans side of the membrane and involves transfer of Gal onto undecaprenol phosphate. The findings suggest that addition of $\alpha$-Gal represents a key limiting step of SCWP assembly and may control the length of this polymer. 